\title{
Qubit coupled-cluster method: A systematic approach to quantum chemistry on a quantum computer
}

\author{
Ilya G. Ryabinkin and Tzu-Ching Yen \\ Department of Physical and Environmental Sciences, \\ University of Toronto Scarborough, Toronto, Ontario M1C1A4, Canada and \\ Chemical Physics Theory Group, Department of Chemistry, \\ University of Toronto, Toronto, Ontario M5S 3H6, Canada \\ Scott N. Genin \\ OTI Lumionics Inc., 100 College St. \#351, Toronto, Ontario M5G 1L5, Canada \\ Artur F. Izmaylov \\ Department of Physical and Environmental Sciences, \\ University of Toronto Scarborough, Toronto, Ontario M1C 1A4, Canada and \\ Chemical Physics Theory Group, Department of Chemistry, \\ University of Toronto, Toronto, Ontario M5S 3H6, Canada
}

(Dated: September 12, 2018)

\begin{abstract}
A unitary coupled-cluster (UCC) form for the wavefunction in the variational quantum eigensolver has been suggested as a systematic way to go beyond the mean-field approximation and include electron correlation in solving quantum chemistry problems on a quantum computer. Although being exact in the limit of including all possible coupled-cluster excitations, practically, the accuracy of this approach depends on how many and what kind of terms are included in the wavefunction parametrization. Another difficulty of UCC is a growth of the number of simultaneously entangled qubits even at the fixed fermionic excitation rank. Not all quantum computing architectures can cope with this growth. To address both problems we introduce a qubit coupled-cluster (QCC) method that starts directly in the qubit space and uses energy response estimates for ranking the importance of individual entanglers for the variational energy minimization. Also, we provide an exact factorization of a unitary rotation of more than two qubits to a product of two-qubit unitary rotations. Thus, the QCC method with the factorization technique can be limited to only two-qubit entanglement gates and allows for very efficient use of quantum resources in terms of the number of coupled-cluster operators. The method performance is illustrated by calculating ground-state potential energy curves of $\mathrm{H}_{2}$ and $\mathrm{LiH}$ molecules with chemical accuracy, $\leq 1 \mathrm{kcal} / \mathrm{mol}$.
\end{abstract}

\section{INTRODUCTION}

A many-body fermionic problem defining electronic properties of molecules and materials from first-principles can be written as the following parametric timeindependent Schrödinger equation

$$
\hat{H}_{e}(\mathbf{R})\left|\Psi_{i}(\mathbf{R})\right\rangle=E_{i}(\mathbf{R})\left|\Psi_{i}(\mathbf{R})\right\rangle .
$$

Here, $\mathbf{R}=\left(\mathbf{R}_{1}, \ldots \mathbf{R}_{N_{n}}\right)$ are nuclear variables treated as parameters, $E_{i}(\mathbf{R})$ and $\left|\Psi_{i}(\mathbf{R})\right\rangle$ are potential energy surfaces (PESs) and adiabatic electronic states, respectively, and $\hat{H}_{e}(\mathbf{R})$ is the electronic Hamiltonian of the system

$$
\begin{aligned}
\hat{H}_{e}(\mathbf{R}) & =-\frac{1}{2} \sum_{i=1}^{N_{e}} \nabla_{\mathbf{r}_{i}}^{2}+\sum_{i<j}^{N_{e}} \frac{1}{\left|\mathbf{r}_{i}-\mathbf{r}_{j}\right|} \\
& +\sum_{\alpha<\beta}^{N_{n}} \frac{1}{\left|\mathbf{R}_{\alpha}-\mathbf{R}_{\beta}\right|}-\sum_{i, \alpha} \frac{1}{\left|\mathbf{r}_{i}-\mathbf{R}_{\beta}\right|},
\end{aligned}
$$

where $\mathbf{r}_{i}$ are the electronic variables, $N_{e}$ and $N_{n}$ are number of electrons and nuclei. Computational cost of the exact numerical solution of Eq. (1) scales exponentially with the size (e.g. the number of electrons) of the system on a classical computer. To address this issue without introducing approximations, it was proposed to employ a quantum computer, ${ }^{1,2}$ which may solve the problem with polynomial scaling of computational cost with the system size.

However, immediate application of quantum computers is hampered by the limited quantity and quality of available quantum resources. In particular, contemporary architectures offer only few $(<10)$ fully interacting qubits, and their quantum coherent state can exist for limited time. As can be gauged both from theoretical and experimental efforts, scaling-up the quantum computer is far from straightforward. Even anticipating noticeable growth of capabilities of quantum hardware in near future, to become competitive with solving the electronic structure problem on classical computers, it is crucial to develop computationally frugal algorithms utilizing present and near future quantum computers in full.

Several algorithmic developments in this direction have already taken place. In order to formulate the electronic structure problem for a quantum computer that operates with qubits (two-level systems), the electronic Hamiltonian needs to be transformed iso-spectrally to its qubit form. This is done in two steps. First, the second quan- 
tized form of $\hat{H}_{e}$ is obtained

$$
\hat{H}_{e}=\sum_{p q} h_{p q}(\mathbf{R}) \hat{a}_{p}^{\dagger} \hat{a}_{q}+\frac{1}{2} \sum_{p q r s} g_{p q r s}(\mathbf{R}) \hat{a}_{p}^{\dagger} \hat{a}_{q}^{\dagger} \hat{a}_{s} \hat{a}_{r}
$$

where $\hat{a}_{p}^{\dagger}\left(\hat{a}_{p}\right)$ are fermionic creation (annihilation) operators, $h_{p q}(\mathbf{R})$ and $g_{p q r s}(\mathbf{R})$ are one- and two-electron integrals in a spin-orbital basis. ${ }^{3}$ This step has polynomial complexity and is carried out on a classical computer. Then, using the Jordan-Wigner $(\mathrm{JW})^{2,4}$ or more resource-efficient Bravyi-Kitaev (BK) transformation, ${ }^{5-9}$ the electronic Hamiltonian is converted iso-spectrally to a qubit form

$$
\hat{H}=\sum_{I} C_{I}(\mathbf{R}) \hat{P}_{I}
$$

where $C_{I}(\mathbf{R})$ are numerical coefficients, and $\hat{P}_{I}$ are Pauli "words", products of Pauli operators of different qubits

$$
\hat{P}_{I}=\cdots \hat{w}_{1}^{(I)} \hat{w}_{0}^{(I)},
$$

$\hat{w}_{i}^{(I)}$ is one of the $\hat{x}, \hat{y}, \hat{z}$ Pauli operators for the $i^{\text {th }}$ qubit.

The quantum phase estimation (QPE) algorithm ${ }^{2,10,11}$ was historically the first approach that relied on estimating the phase of the stationary-state wavefunction using the quantum Fourier transform. ${ }^{12}$ For good accuracy one would need to perform a long coherent evolution of the system for accurate phase estimation. Practical difficulties in maintaining qubits' coherence for long time hinder the use of the QPE approach for quantum chemistry simulations on currently available quantum computers.

This prompted a development of a more resourceefficient method, the variational quantum eigensolver (VQE), ${ }^{13,14}$ where the trial ground-state stationary wavefunction $\Psi$ is prepared by an action of a suitably chosen unitary operator $\hat{U}$ on an initial state of qubits $|\Phi\rangle$, $|\Psi\rangle=\hat{U}|\Phi\rangle . \hat{U}$ is optimized variationally to provide an estimate for the ground state exact energy

$$
E_{0} \leq\langle\hat{U} \Phi|\hat{H}| \hat{U} \Phi\rangle .
$$

Of course, there is a great deal of freedom in parametrization of $\hat{U}$. The original proposal of Ref. 13 was to borrow the parametrization of $U$ from the fermion-to-qubit transformation of a fermionic unitary coupled-cluster (UCC) ansatz,

$$
\hat{U}_{\mathrm{CC}}=\exp \left(\hat{T}-\hat{T}^{\dagger}\right),
$$

where

$$
\begin{aligned}
\hat{T} & =\hat{T}_{1}+\hat{T}_{2}+\ldots+\hat{T}_{N_{e}}, \\
\hat{T}_{1} & =\sum_{i a} t_{i}^{a} \hat{a}_{a}^{\dagger} \hat{a}_{i}, \\
\hat{T}_{2} & =\sum_{i j a b} t_{i j}^{a b} \hat{a}_{a}^{\dagger} \hat{a}_{b}^{\dagger} \hat{a}_{j} \hat{a}_{i},
\end{aligned}
$$

Here $\hat{T}_{k}$ are mutually commuting fermionic $k$-fold (single, double, etc.) excitation operators that promote electrons from occupied orbitals $i, j, \ldots$ to unoccupied (virtual) orbitals $a, b, \ldots$ in a reference single-determinant $|\Phi\rangle$ and parametrized by the corresponding amplitudes $t_{i}^{a}, t_{i j}^{a b}, \ldots$ $\hat{T}$ may be truncated at rank $m \leq N_{e}$, leading to an approximate treatment, but at any rank greater than 1 the UCC ansatz is exponentially hard for a classical computer due to non-truncation of the Baker-Campbell-Hausdorff series. ${ }^{15}$ On the other hand, it is known that coupled cluster-type expansions are quickly convergent, especially if electron correlation is not too strong, ${ }^{16}$ thus, there is a hope to find accurate energy estimates already at the low rank.

Yet, even though superiority of the UCC-based VQE approach over QPE has been convincingly demonstrated, ${ }^{17}$ the computational cost of UCC still grows relatively quickly even at low orders. The main difficulty is that all fermion-to-qubit transformations increase the length of Pauli words involved in $\hat{U}$. In other words, even if only a one-body cluster operator, $\hat{T}_{1}$, is used for $\hat{U}_{\mathrm{CC}}$, its qubit representation is a unitary operator involving Pauli words of the length $\sim \log \left(N_{o}\right)$ (in the case of the BK transformation, others increase the length even faster), where $N_{o}$ is the number of one-electron spinorbitals in the second quantized form of $\hat{H}_{e}$. For some lattice models there are special transformations that limits this growth of locality,${ }^{9}$ but for a general molecular Hamiltonian the best count is still logarithmic. This becomes a challenge because multi-qubit operations require either entangling of increasing number of qubits (not all quantum computers can do that) or factorizing the multiqubit operation as a product of one- and two-qubit ones. The latter was shown to be possible in principle, but there are no general approaches to construct an efficient factorization proposed to date. ${ }^{12}$ A typical approximate approach involves Trotterization. ${ }^{18}$ Frequently, fermionic excitation operators, Eqs. (9-10), are transformed to a qubit form and Trotterized. ${ }^{19,20}$

Another strategy for constructing the wavefunction has been adopted recently in Ref. 21. The wavefunction ansatz was tailored to hardware requirements and had the form

$$
\begin{aligned}
\Psi(\boldsymbol{\theta})= & \prod_{q=1}^{N_{q}}\left[U^{q, d}(\boldsymbol{\theta})\right] \times U_{\mathrm{ENT}} \times \cdots \\
& \times \prod_{q=1}^{N_{q}}\left[U^{q, 1}(\boldsymbol{\theta})\right] \times U_{\mathrm{ENT}} \times \prod_{q=1}^{N_{q}}\left[U^{q, 0}(\boldsymbol{\theta})\right]|00 \ldots 0\rangle,
\end{aligned}
$$

where $N_{q}$ is the number of qubits, $|00 \ldots 0\rangle$ is an initial state of the qubits and is exact equivalent of $|\Phi\rangle$ in Eq. (6). Equation (11) is an alternating sequence of products of individual qubit rotations $U^{q, j}(\boldsymbol{\theta})$,

$$
U^{q, j}(\boldsymbol{\theta})=\mathrm{e}^{i z_{q} \theta_{1}^{q, j}} \mathrm{e}^{i x_{q} \theta_{2}^{q, j}} \mathrm{e}^{i z_{q} \theta_{3}^{q, j}}, 0 \leq j \leq d
$$


where $\theta_{1}^{q, j}, \theta_{2}^{q, j}$, and $\theta_{3}^{q, j}$ are the Euler angles of the $q^{\text {th }}$ qubit at the $j^{\text {th }}$ level, interleaved with action of "entanglers"

$$
U_{\mathrm{ENT}}(\tau)=\exp (-i \tau \hat{P}),
$$

where $\tau$ is an amplitude, and $\hat{P}$ is a two-qubit Pauli word. The number $d$ was called a depth of the scheme. The first set of $z$-rotations in $U^{q, 0}(\boldsymbol{\theta})$ was not implemented $\left(\theta_{3}^{q, 0}=0, q=1 \ldots N_{q}\right)$, and entanglers' amplitudes $\tau$ were all kept fixed, so that the ansatz (11) contained $N_{q}(3 d+2)$ variational parameters in total. By minimizing the expectation value of the Hamiltonian, Eq. (6), with respect to these parameters for sufficiently high depths, reasonably accurate ground-state electronic energies as functions of internuclear distances for molecules $\mathrm{H}_{2}, \mathrm{LiH}$, and $\mathrm{BeH}_{2}$ were obtained.

The obvious advantage of the form (11) is its minimal degree of entanglement, only two qubits at a time. The unitary transformation is given directly in the qubit space, in contrast to the UCC form that starts with fermionic operators. Perhaps, this fact alone is responsible for considerable reduction of quantum resources needed for simulations and eventually allowed for successful simulations of molecules larger than $\mathrm{H}_{2}$. The rigorous basis for convergence of ansatz (11) to the exact answer with increasing depth $d$ is the theorem stating that any multi-qubit unitary transformation can be presented as a product of one- and two-qubit unitary transformations. ${ }^{22}$ However, efficient construction of such a factorization is yet unsolved problem. Therefore, it is clear that the form (11) can be improved if some specifics of the problem is taken into account. First, it can be shown that only two individual qubit rotation angles per each qubit are sufficient at any depth $d$, provided that entanglers' amplitudes are fully optimized. Second, a set of pre-defined generators of entanglement $\hat{P}$ [Eq. (13)] may not lead to the fastest convergence - it is the system which defines the most "efficient" entanglers, not the hardware.

In the current paper we introduce a new systematic approach, a qubit coupled-cluster (QCC) method, within the VQE formalism. QCC resembles a coupled-cluster hierarchy, but contrary to UCC, it is built directly in the qubit space. Therefore, we bypass any explicit fermionic constructions and save as much quantum resources as possible. To achieve systematic convergence, we introduce an entanglers' screening protocol that ranks various entanglers according to their estimated contributions into the correlation energy. To allow for an arbitrary multiqubit entanglers on limited-qubit hardware, we derive an exact factorization formula that represents a multi-qubit entangler as a product of two-qubit entanglers. This factorization uses commutation relations of the qubit Lie algebra and introduce the minimal number of terms. We illustrate its essential features on ground potential energy surfaces of the $\mathrm{H}_{2}$ and $\mathrm{LiH}$ molecules, which are small enough to be amenable for a comprehensive study, while at the same time are complex enough to illustrate many quantum-chemical phenomena, including the transition to the strong-correlation limit upon dissociation.

\section{THEORY}

\section{A. Qubit Coupled-Cluster method}

The QCC wavefunction has the form

$$
\Psi(\boldsymbol{\tau}, \boldsymbol{\Omega})=\hat{U}(\boldsymbol{\tau})|\boldsymbol{\Omega}\rangle,
$$

where $|\boldsymbol{\Omega}\rangle$ and $\hat{U}(\boldsymbol{\tau})$ represent the mean-field and correlation parts of a wavefunction. The mean-field wavefunction is a product of single-qubit coherent states $^{23-26}$

$$
\begin{aligned}
|\boldsymbol{\Omega}\rangle & =\prod_{i=1}^{N_{q}}\left|\Omega_{i}\right\rangle, \\
\left|\Omega_{i}\right\rangle & =\cos \left(\frac{\theta_{i}}{2}\right)|\alpha\rangle+\mathrm{e}^{\mathrm{i} \phi_{i}} \sin \left(\frac{\theta_{i}}{2}\right)|\beta\rangle,
\end{aligned}
$$

where $\phi_{i}$ and $\theta_{i}$ are azimuthal and polar angles on the "Bloch sphere" of the $i^{t h}$ qubit, respectively, and $|\alpha\rangle$ and $|\beta\rangle$ are spin-up and spin-down eigenstates of the $\hat{s}_{z}(i)=\hat{z}_{i} / 2$ operator. The single-qubit coherent states constitute a normalized but non-orthogonal complete set. Correlation is introduced by multi-qubit rotations parametrized by real-valued amplitudes $\boldsymbol{\tau}=\left\{\tau_{k}\right\}$ as

$$
\hat{U}(\boldsymbol{\tau})=\prod_{k=1}^{N_{\mathrm{ent}}} \exp \left(-i \tau_{k} \hat{P}_{k} / 2\right),
$$

where $\hat{P}_{k}$ are the Pauli words [Eq. (5)] whose lengths vary from 2 to $N_{q}, N_{\text {ent }}$ is less or equal to the total number of possible $\hat{P}_{k}$ operators, $4^{N_{q}}-3 N_{q}-1$, and a factor of $1 / 2$ is introduced for convenience.

The expectation value of the Hamiltonian for the QCC parametrization [Eq. (14)] is

$$
\begin{aligned}
E(\boldsymbol{\tau}, \boldsymbol{\Omega}) & =\langle\Psi(\boldsymbol{\tau}, \boldsymbol{\Omega})|\hat{H}| \Psi(\boldsymbol{\tau}, \boldsymbol{\Omega})\rangle \\
& =\left\langle\boldsymbol{\Omega}\left|U(\boldsymbol{\tau})^{\dagger} \hat{H} U(\boldsymbol{\tau})\right| \boldsymbol{\Omega}\right\rangle,
\end{aligned}
$$

and minimization of $E(\boldsymbol{\tau}, \boldsymbol{\Omega})$ with respect to amplitudes $\tau$ and angles in $\boldsymbol{\Omega}$ provides the ground state energy. The main difference between QCC and UCC forms is in the nature of generators of unitary transformations. In the UCC formalism generators are sums of mutuallycommuting fermionic operators [Eqs (8-10)], each of those, in turn, after the JW or BK transformation is a lengthy linear combination of Pauli words. This leads to excessive consumption of precious quantum resources, for example, two-particle entangling gates. Moreover, exponent of such quantities are not known in closed form and, thus, are not amenable for extensive analysis. In contrast, the $\hat{P}_{k}$ generators of the QCC ansatz are simple Pauli words, and thus, all of them are involutory operators, $\hat{P}_{k}^{2}=1$. This property provides a closed form for 
similarity transformed Hamiltonians of individual entanglers

$$
\begin{aligned}
\hat{H}[\tau ; \hat{P}] & =\mathrm{e}^{\mathrm{i} \tau \hat{P} / 2} \hat{H} \mathrm{e}^{-\mathrm{i} \tau \hat{P} / 2} \\
& =\hat{H}-\mathrm{i} \frac{\sin \tau}{2}[\hat{H}, \hat{P}]+\frac{1}{2}(1-\cos \tau) \hat{P}[\hat{H}, \hat{P}] .
\end{aligned}
$$

Hence, it is obvious that the fully transformed Hamiltonian $\hat{H}=U(\boldsymbol{\tau})^{\dagger} \hat{H} U(\boldsymbol{\tau})$ in Eq. (19) will involve $\sim 3^{N_{\text {ent }}}$ operators thus revealing the exponential complexity of the QCC form.

Overall, the QCC scheme requires $2 N_{q}+N_{\text {ent }}$ variational parameters, where $2 N_{q}$ is the number of Bloch angles for $N_{q}$ qubits, and $N_{\text {ent }}$ is the number of optimized entanglers' amplitudes.

One possible concern about using Pauli words as generators is potential breaking of the fermionic symmetry by a trial electronic function and ensuing unphysical behavior of energy an properties. Indeed, an approximated wavefunction is prone to such a phenomenon, ${ }^{27}$ and as we found in Ref. 28 , it is more a rule than an exception. However, the QCC formalism can be equipped with constraints, such as the particle number or electronic spin, in a straightforward manner to guarantee conservation of proper physical symmetries.

\section{B. Entanglers' ranking}

The key quantity in our ranking of all, $4^{N_{q}}-3 N_{q}-$ 1 entanglers for the $N_{q}$-qubit system is the similaritytransformed energy function,

$$
E\left[\tau ; \hat{P}_{k}\right]=\min _{\boldsymbol{\Omega}}\left\langle\boldsymbol{\Omega}\left|e^{i \tau \hat{P}_{k} / 2} \hat{H} e^{-i \tau \hat{P}_{k} / 2}\right| \boldsymbol{\Omega}\right\rangle .
$$

Using the closed form of the similarity transformed Hamiltonian, Eq. (20), it is straightforward to evaluate $E\left[\tau ; \hat{P}_{k}\right]$ and to find the energy lowering due to variation of $\tau$

$$
\begin{aligned}
\Delta E\left[\hat{P}_{k}\right] & =\min _{\tau} E\left[\tau ; \hat{P}_{k}\right]-E\left[0 ; \hat{P}_{k}\right] \\
& =\min _{\tau} E\left[\tau ; \hat{P}_{k}\right]-E_{\mathrm{QMF}} \leq 0 .
\end{aligned}
$$

Here $E_{\mathrm{QMF}}=\min _{\boldsymbol{\Omega}}\langle\boldsymbol{\Omega}|\hat{H}| \boldsymbol{\Omega}\rangle$ is the qubit mean-field (QMF) energy, which is equivalent to $E\left[0 ; \hat{P}_{k}\right]$. Thus, all entanglers can be ranked according to their $\Delta E\left[\hat{P}_{k}\right]$ values. The main downside of this procedure is its computational cost: it requires full optimization of both $\tau$ and the mean-field angles $\boldsymbol{\Omega}$ for each entangler that can be expensive for large systems.

To alleviate this problem, a pre-screening based on the first and second terms of the Taylor expansion of individual similarity-transformed energies [Eq. (21)]

$$
\begin{aligned}
E\left[\tau ; \hat{P}_{k}\right]=E_{\mathrm{QMF}} & +\left.\tau \frac{\mathrm{d} E\left[\tau ; \hat{P}_{k}\right]}{\mathrm{d} \tau}\right|_{\tau=0} \\
& +\left.\frac{\tau^{2}}{2} \frac{\mathrm{d}^{2} E\left[\tau ; \hat{P}_{k}\right]}{\mathrm{d} \tau^{2}}\right|_{\tau=0}+\ldots
\end{aligned}
$$

can be done. One caveat in calculating these derivatives is accounting for the relaxation of the mean-field part due to changes in $\tau$. Essentially, for every value of $\tau$ the minimizing set of Bloch angles is different, therefore they are implicit functions $\tau$. Thus, the full $\tau$ derivative in Eq. (23) can be expanded as

$$
\frac{\mathrm{d}}{\mathrm{d} \tau}=\frac{\partial}{\partial \tau}+\sum_{i=1}^{2 N_{q}}\left(\frac{\mathrm{d} \varphi_{i}}{\mathrm{~d} \tau}\right)_{\min } \frac{\partial}{\partial \varphi_{i}}
$$

where $\left\{\varphi_{i}\right\}_{i=1}^{2 N_{q}}$ denotes $\left\{\theta_{1}, \phi_{1}, \ldots \theta_{N_{q}}, \phi_{N_{q}},\right\}$ the set of the Bloch angles parametrizing the mean-field solution. It is straightforward to show (see the Appendix) that the first derivative at $\tau=0$ is given by:

$$
\left.\frac{\mathrm{d} E\left[\tau ; \hat{P}_{k}\right]}{\mathrm{d} \tau}\right|_{\tau=0}=\left\langle\boldsymbol{\Omega}_{\min }\left|-\frac{\mathrm{i}}{2}\left[\hat{H}, \hat{P}_{k}\right]\right| \boldsymbol{\Omega}_{\min }\right\rangle,
$$

where $\left|\boldsymbol{\Omega}_{\min }\right\rangle$ is a coherent state evaluated on the set of optimized at the mean-field level angles $\left\{\varphi_{i}\right\}$. Evaluation of the second order derivative in Eq. (23) is more involved and is detailed in the Appendix.

Thus, the important entanglers can be ranked within two tiers. The first tier is formed by the entanglers with non-zero absolute values of their first energy derivatives [Eq. (25)]. The second tier includes the entanglers with vanishing first energy derivatives but significant negative second energy derivatives, Eq. (A.12). The final ranking is done based on evaluating $\Delta E\left[\hat{P}_{k}\right]$ values for top entanglers in both tiers.

\section{Factorization of multi-qubit entanglers}

Existing quantum hardware is typically limited in the form of operators it can efficiently implement. One of such limitations is the maximum number of qubits that are possible to entangle by realizable unitary transformations, in other words, the length of the Pauli word [Eq. (5)] generating a unitary transformation is usually limited to two qubits. Thus, if our ranking procedure identifies three- or four-body entanglers as contributing significantly to the energy lowering, it may not be possible to directly implement them due to hardware limitations.

Here, we show how to factorize a unitary transformation entangling more than two qubits in a product of unitary transformations involving only two-qubit generators. The factorization is a recursive procedure applied at each step to a many-qubit unitary to produce three new unitaries, each of them parametrized by fewer-qubits Pauli words.

The elementary factorization step can be illustrated as follows:

1. Assume we have Pauli word $\hat{P}$ with the length $|\hat{P}| \geq 3$, where the length $|\cdot|$ is defined as the 
number of Pauli operators in $\hat{P}$. Then $\hat{P}$ can be represented as

$$
\hat{P}=\hat{P}_{1} \hat{w}_{k} \hat{P}_{2},
$$

where the subscript of $\hat{w}_{k}$ denotes that this elementary Pauli operator corresponds to the $k^{\text {th }}$ qubit. The word $\hat{P}_{1}\left(\hat{P}_{2}\right)$ contains all qubit indices that are strictly greater (lower) than $k . \hat{P}_{1}, \hat{P}_{2}$, and $\hat{w}_{k}$ are all mutually commutative, since they act on non-overlapping sets of qubits. Also by a choice of $k$ one can always satisfy the following relations

$\left|\hat{P}_{1,2}\right|= \begin{cases}\frac{|\hat{P}|-1}{2}, & |\hat{P}| \text { is odd } \\ \frac{|\hat{P}|}{2} \text { and } \frac{|\hat{P}|}{2}-1, & |\hat{P}| \text { is even }\end{cases}$

2. Let us denote $\hat{w}_{k}^{\prime}$ and $\hat{w}_{k}^{\prime \prime}$ the Pauli operators for the $k^{\text {th }}$ qubit that satisfy the following commutation relation with $\hat{w}_{k}$

$$
\left[\hat{w}_{k}^{\prime}, \hat{w}_{k}^{\prime \prime}\right]=2 \mathrm{i} \hat{w}_{k},
$$

which is always possible by properties of a singlequbit Pauli operators. Substituting $\hat{w}_{k}$ by its commutator in Eq. (26) gives

$$
\hat{P}=-\frac{i}{2}\left[\hat{P}_{1} \hat{w}_{k}^{\prime}, \hat{w}_{k}^{\prime \prime} \hat{P}_{2}\right] .
$$

On the other hand, the same commutator as in Eq. (29) can be obtained if the expression $\exp \left[\mathrm{i}(\pi / 4) \hat{w}_{k}^{\prime \prime} \hat{P}_{2}\right] \hat{P}_{1} \hat{w}_{k}^{\prime} \exp \left[-\mathrm{i}(\pi / 4) \hat{w}_{k}^{\prime \prime} \hat{P}_{2}\right]$ is considered using Eq. (20), and therefore

$$
\hat{P}=\mathrm{e}^{\mathrm{i}(\pi / 4) \hat{w}_{k}^{\prime \prime} \hat{P}_{2}} \hat{P}_{1} \hat{w}_{k}^{\prime} \mathrm{e}^{-\mathrm{i}(\pi / 4) \hat{w}_{k}^{\prime \prime} \hat{P}_{2}} .
$$

3. Finally, from Eq. (30) it follows that

$$
\hat{P}^{n}=\mathrm{e}^{\mathrm{i}(\pi / 4) \hat{w}_{k}^{\prime \prime} \hat{P}_{2}}\left(\hat{P}_{1} \hat{w}_{k}^{\prime}\right)^{n} \mathrm{e}^{-\mathrm{i}(\pi / 4) \hat{w}_{k}^{\prime \prime} \hat{P}_{2}},
$$

and therefore, exponentiation of $\hat{P}$ using the Taylor series gives the final factorization

$$
\mathrm{e}^{-\mathrm{i} t \hat{P}}=\mathrm{e}^{\mathrm{i}(\pi / 4) \hat{w}_{k}^{\prime \prime} \hat{P}_{2}} \mathrm{e}^{-\mathrm{i} t \hat{P}_{1} \hat{w}_{k}^{\prime}} \mathrm{e}^{-\mathrm{i}(\pi / 4) \hat{w}_{k}^{\prime \prime} \hat{P}_{2}} .
$$

Eq. (32) expresses the unitary transformation with the generator $\hat{P}$ as a product of three unitary transformations with generators of at most $\lfloor|\hat{P}| / 2\rfloor+1$ length. Thus, the number of two-qubit factors grows as $\sim \log _{2}|\hat{P}|$. Also, note that the factorization does not increase the number of variational parameters, only the number of entanglers.

\section{NUMERICAL STUDIES AND DISCUSSION}

We illustrate our developments by computing the ground state potential energy curves within the QMF and QCC approaches for the $\mathrm{H}_{2}$ and $\mathrm{LiH}$ molecules. These molecules were used to illustrate performance of quantum computing techniques previously. ${ }^{21,29} \mathrm{LiH}$ is particular interesting because it is one of the simplest molecules, where electronic correlation entangles electrons on more than two orbitals.

\section{A. Fermionic Hamiltonian quantities}

To generate fermionic Hamiltonian spin-orbitals and one- and two-electron integrals, a locally modified GAMESS $^{30}$ electronic structure package was used.

Molecules were oriented along the $z$ axis, and canonical restricted Hartree-Fock (RHF) orbitals were computed in the STO-3G atomic basis set ${ }^{31}$ assuming $D_{2 h}$ and $C_{2 v}$ symmetry for $\mathrm{H}_{2}$ and $\mathrm{LiH}$, respectively. ${ }^{32}$ For the $\mathrm{H}_{2}$ molecule all 4 spin-orbitals were taken into account in construction of the second-quantized electronic Hamiltonian, Eq. (3). For the LiH molecule we determine the subset of "active" spin-orbitals to produce the electronic Hamiltonian as follows. Out of 6 molecular orbitals of $\mathrm{LiH}$, four belong to $A_{1}$ and two remaining correspond to the $B_{1}$ and $B_{2}$ irreducible representations of the $C_{2 v}$ group. The lowest-energy $A_{1}$-type orbital and both $B$ type orbitals were frozen, which means their populations were fixed at 2,0 , and 0 , respectively. The remaining 3 orbitals of $A_{1}$ type constituted the active space where the electronic Hamiltonian (3) was computed. It must be noted that one of $A_{1}$ orbitals lies higher in energy than both $B$-type ones, so that reordering of the canonical RHF orbitals, which are typically sorted by orbital energy, was necessary. Molecular integral transformation was carried out by the ALDET module; this module was also used to generate reference complete active space configurational interaction (CASCI) energies.

\section{B. Generation of qubit operators}

$\mathrm{H}_{2}$ molecule: One- and two-electron integrals in the canonical RHF molecular orbitals basis for each value of the internuclear distance, $R(\mathrm{H}-\mathrm{H})$, were used in the BK transformation to produce the corresponding qubit Hamiltonians. Spin-orbitals were alternating in the order $\alpha, \beta, \alpha, \ldots$ Although qubits 2 and 4 are known to be stationary, in other words, all operators in the qubit Hamiltonian act by only $z$ or the identity operators on these qubits, we did not use this additional symmetry to decrease the qubit count in the qubit Hamiltonians, so that all 4 qubits corresponding to 4 spin-orbitals were retained. The number of electrons, $\hat{N}$, and the square of the total spin, $\hat{S}^{2}$, operators were prepared from their second quantized fermionic expressions. ${ }^{3}$ These operators are extremely useful in analysis of both QMF and QCC solutions.

LiH molecule: Qubit Hamiltonians for various $R(\mathrm{Li}-$ $\mathrm{H})$ distances were generated using the parity fermion-toqubit transformation. ${ }^{33}$ Spin-orbitals were arranged as "first all alpha then all beta" in the fermionic form; since there are 3 active molecular orbitals in the problem, this lead to a 6-qubit Hamiltonian containing 118 Pauli words for each $R(\mathrm{Li}-\mathrm{H})$. These qubit Hamiltonians have $3^{\text {rd }}$ and $6^{\text {th }}$ stationary qubits, which allows one to replace the corresponding $\hat{z}$ operators by their eigenvalues, \pm 1 , thus defining the different "sectors" of the original Hamilto- 
TABLE I. Two-qubit entangler ranking for $\mathrm{H}_{2}$ at $R=1.0 \AA$ by Eqs. (25), (A.12), and (22). Hartree atomic units.

\begin{tabular}{lrrr}
\hline Entangler $\hat{P}$ & $\left.\left.\frac{\mathrm{d} E(\tau ; \hat{P})}{\mathrm{d} \tau}\right|_{\tau=0} \frac{\mathrm{d}^{2} E(\tau ; \hat{P})}{\mathrm{d} \tau^{2}}\right|_{\tau=0}$ & $\Delta E[P]$ \\
\hline$\hat{x}_{2} \hat{y}_{0}$ & -0.1968 & 0.5343 & -0.0350 \\
$\hat{y}_{2} \hat{x}_{0}$ & 0.1968 & 0.5343 & -0.0350 \\
$\hat{z}_{2} \hat{y}_{0}$ & 0.0000 & -0.0509 & -0.0350 \\
$\hat{z}_{2} \hat{x}_{0}$ & 0.0000 & -0.0509 & -0.0350 \\
$\hat{y}_{2} \hat{z}_{0}$ & 0.0000 & -0.0493 & -0.0350 \\
$\hat{x}_{2} \hat{z}_{0}$ & 0.0000 & -0.0493 & -0.0350 \\
\hline
\end{tabular}

nian. Each of these sectors is characterized by its own 4-qubit effective Hamiltonian. The ground state lies in the $z_{2}=-1, z_{5}=1$ (qubits are enumerated from 0 ) sector; the corresponding effective Hamiltonian has 100 Pauli terms. Analogous reduction procedure has been applied to the qubit images of $\hat{N}$ and $\hat{S}^{2}$ operators.

\section{Potential energy curves}

As we established in Ref. 28, variational methods in qubit space are prone to symmetry breaking. This is particularly dramatically seeing in the QMF case (Figs. 1 and 3 ). To restore the correct singlet spin symmetry and to make PESs smooth, it is enough to imposed the spin constraint $\left(\left\langle S^{2}\right\rangle=0\right)$ on the QMF solutions. Alternatively, more correlation can be added to QMF to approach the exact solution, which has the correct singlet symmetry. In all our QCC calculations we started with symmetry unconstraint QMF solutions.

Entanglers in $\mathrm{H}_{2}$ : We rank entanglers at $R(\mathrm{H}-\mathrm{H})$ $=1.0 \AA$ according to the procedure described in Sec. II B (the full table can be found in Supplementary materials). Since only two out of four qubits contribute nontrivially to the eigenstates of the $\mathrm{H}_{2}$ qubit Hamiltonian, two-qubit entanglers are sufficient for obtaining the exact solution. Out of total 54 two-qubit entanglers only 6 provide energy lowering; all of them were found by the combined first- [Eq. (25)] and second-order [Eq. (A.12)] screening, see Table I. Only 2 of them has non-zero energy gradient and, thus, are identified by the cheapest first-order test, Eq. (25). Moreover, since all 6 energy-lowering entanglers are related by a simple global frame rotation (change of quantization axis) it is possible to use any one of them for the whole range of $R(\mathrm{H}-\mathrm{H})$, see Fig. 1. Thus, the $\mathrm{H}_{2}$ molecule in the minimal basis requires only one entangler to reach the exact energy. This entangler is readily identified by our ranking procedure without any ad hoc assumptions about the structure of a wave function or reference to fermionic operators. A quantum computer circuit suitable for the Rigetti machine ${ }^{34}$ that solves the $\mathrm{H}_{2}$ problem by the QCC method with the chosen entangler is shown in Fig. 2. Besides a set of one-qubit gates, such as rotations around $x(\mathrm{RX})$ and $z(\mathrm{RZ})$ axis, and the Hadamard $(\mathrm{H})$ gate, it contains only two two-qubit CNOT gates. They appear as a result of a decomposition
TABLE II. Top entanglers for LiH at $R=3.2 \AA$ by Eqs. (25), (A.12), and (22). Hartree atomic units. $\Delta E[P]$ 's are evaluated with respect to the symmetry constrained QMF energies.

\begin{tabular}{lcc}
\hline Entangler $\hat{P}$ & $\mathrm{~d} E_{\mathrm{cQMF}}(\tau ; \hat{P})$ & \\
\cline { 2 - 3 }$\hat{x}_{2} \hat{x}_{1} \hat{y}_{0}$ & -0.0792 & $\Delta E[P]$ \\
$\hat{z}_{3} \hat{y}_{2} \hat{z}_{1} \hat{x}_{0}$ & -0.0781 & -0.0909 \\
$\hat{x}_{2} \hat{y}_{1} \hat{x}_{0}$ & -0.0792 & -0.0909 \\
$\hat{z}_{3} \hat{x}_{2} \hat{x}_{1} \hat{y}_{0}$ & -0.0792 & -0.0896 \\
$\hat{x}_{3} \hat{y}_{2} \hat{z}_{1} \hat{x}_{0}$ & -0.0792 & -0.0896 \\
$\hat{z}_{3} \hat{x}_{2} \hat{y}_{1} \hat{x}_{0}$ & -0.0792 & -0.0887 \\
$\hat{y}_{3} \hat{x}_{2} \hat{z}_{1} \hat{x}_{0}$ & -0.0792 & -0.0887 \\
\hline
\end{tabular}

(made by PYQuiL ${ }^{35}$ ) of the unitary transformation generated by the entangler $\hat{x}_{2} \hat{y}_{0}$ into elementary operations possible on the Rigetti machine. The two leftmost rows of single-qubit gates encode the spin coherent states and depend on the Bloch angles $\left(\phi_{i}, \theta_{i}\right), i=1, \ldots, 4$, the RZ gate interlaid between the two CNOT gates depends on the entangler's amplitude $\tau$.

Entanglers in LiH: Due to a larger number of entangled spin-orbitals in $\mathrm{LiH}$, the list of entanglers is more complex. Near the equilibrium bond length, $R(\mathrm{Li}-\mathrm{H}) \approx$ $1.5 \AA$, our procedure readily identifies a few (6-7) entanglers that give chemical accuracy. However, these entanglers do not provide chemical accuracy for the whole curve, $R(\mathrm{Li}-\mathrm{H})$ from 0.5 to $5.0 \AA$. The region where $\mathrm{QMF}$ experiences symmetry breaking, $R(\mathrm{Li}-\mathrm{H}) \gtrsim 3.2 \AA$, is especially demanding: the list of energetically-important entanglers grows substantially and calculating the same entanglers' characteristics as before does not shrink it considerably. To achieve reduction, we noticed that since the QMF reference has broken symmetry while the exact eigenstate has the singlet symmetry, it is prudent to search for entanglers that can not only lower the energy but also restore the spin symmetry. To assess the capability of entanglers for symmetry restoration we calculate the gradient of individual energies on a spin-singlet con-

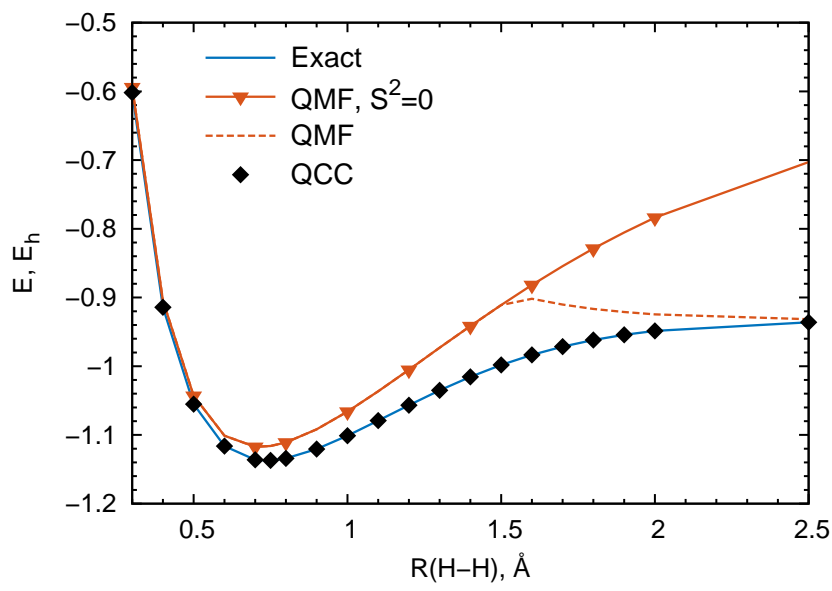

FIG. 1. Potential energy curves for the $\mathrm{H}_{2}$ molecule. 


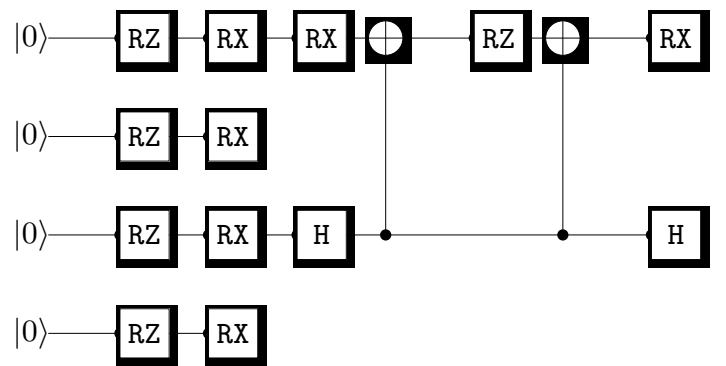

FIG. 2. A sample quantum circuit representing the QCC ansatz for the $\mathrm{H}_{2}$ molecule on the Rigetti machine.

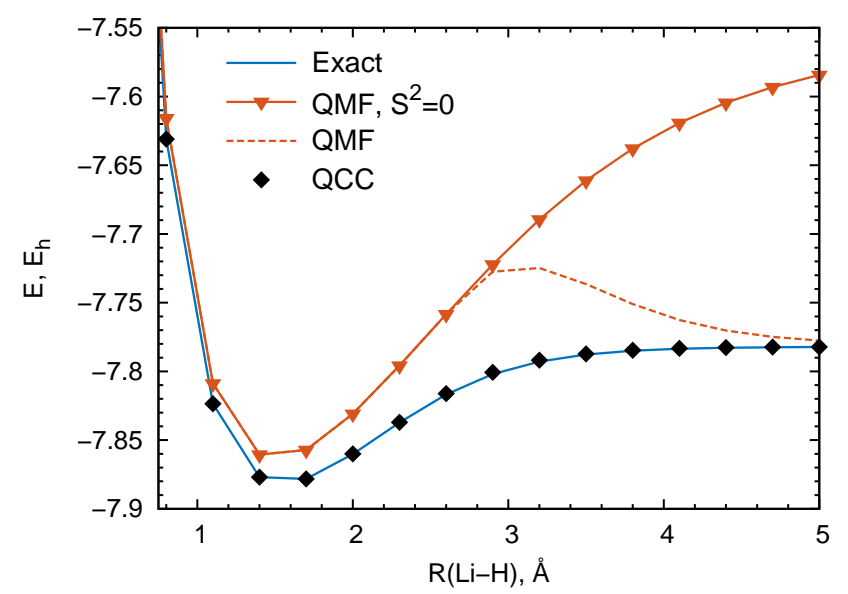

FIG. 3. Potential energy curves for the LiH molecule.

straint $\mathrm{QMF}$ solution, $\left|\boldsymbol{\Omega}_{\mathrm{c}, \min }\right\rangle$ :

$$
\left.\frac{\mathrm{d} E_{\mathrm{cQMF}}\left[\tau ; \hat{P}_{k}\right]}{\mathrm{d} \tau}\right|_{\tau=0}=\left\langle\boldsymbol{\Omega}_{\mathrm{c}, \min }\left|-\frac{\mathrm{i}}{2}\left[\hat{H}, \hat{P}_{k}\right]\right| \boldsymbol{\Omega}_{\mathrm{c}, \min }\right\rangle .
$$

These gradients are larger for those entanglers that can affect the symmetry of the constrained QMF solution. Using 7 of such entanglers, which also have high individual energy lowerings (Table II), we were able to reach the chemical accuracy for the whole $\mathrm{LiH}$ potential energy curve (Fig. 3). Interestingly, these entanglers that can not only restore symmetry but break it, work well for nuclear geometries where symmetry restoration is not needed. We attribute this to ability of the variational procedure to find amplitudes for the symmetry affecting entanglers so that they do not break QMF symmetry where it is unnecessary.

Applying our factorization procedure to split threeand four-qubit entanglers in Table II gives 31 two-qubit entanglers. In general, the decomposition overhead is logarithmic, but the exact figures depend on the set of entanglers. The factorization does not change the number of variational parameters and their total number in the $\mathrm{QCC}$ procedure for $\mathrm{LiH}$ is only $2 \times 4+7=15$.

If we compare our QCC ansatz with alternatives from the literature for the $\mathrm{LiH}$ application, we find the following. If one uses the QPE method, order of 100 exponential factors - the size of the Hamiltonian - are needed.

The simplified UCC ansatz of Ref. 29 started from anal- ysis of fermionic double excitation operators [Eq. (10)] inferred from the configuration-interaction single and double excitation (CISD) calculations. It was found 16 Pauli words are necessary to represent only two most important ones; to be able to proceed the authors kept rather arbitrarily chosen two two-qubit entanglers out of this list.

The form of Kandala et al. ${ }^{21}$ seems to be the most economical one: chemical accuracy was achieved with $d=6$, which implies at least 6 pairwise entanglers. However, Kandala et al. ${ }^{21}$ use significantly more variational parameters: for 4 qubits and $d=6$ the count is 80 . The excessive parametrization makes energy minimization considerably more difficult since the expectation values provided by a quantum computer have inherent quantum uncertainty.

\section{CONCLUSIONS}

We have introduced and studied a new method for performing electronic structure calculations on a quantum computer. We called our method the qubit coupledcluster (QCC) to emphasize that it is rooted in the unitary coupled-cluster (UCC) theory, but contrary to UCC, formulated without any reference to fermionic algebra exclusively in terms of qubit operators.

From the mathematical point of view, QCC is a variational method where variational optimization of singlequbit rotations and multi-qubit unitary transformation are done altogether. The single-qubit Hilbert space is parametrized in terms of spin coherent states which depend on Bloch angles. There are only two angles per qubit, and terms of the qubit Hamiltonian have exceptionally simple expressions for matrix elements in the chosen basis.

The essential feature of the QCC form is the multiqubit unitary transformations in terms of Pauli wordsproducts of Pauli operators. Such transformations are the simplest possible in the qubit tensor algebra; they lead to a compact economical form of unitarily-transformed operators like the Hamiltonian itself [Eq. (20)]. This simplicity allows for extremely frugal use of quantum resources such as two-qubit entanglement gates. Moreover, we derived the decomposition of a multiqubit unitary rotation to a product of two-qubit unitary rotations, Eq. (32). This formula can make simulations of highly entangled systems possible on hardware that is limited by entanglement of only two qubits.

Formulations of the quantum chemistry in the qubit space have several complications. First, even though only iso-spectral fermionic-to-qubit transformations are employed, and thus all symmetries of the fermionic system are replicated in its qubit counterpart, this is not generally true for approximate methods. Indeed, in Ref. 28 we found that breaking of physical symmetries, such as the number of electrons or spin, in qubit variational methods is more a rule than an exception. At the same time, this problem can be solved by using appropriate 
constraints. Second, by treating a molecular system directly in the qubit space, it is not straightforward to use typical electronic structure intuition, for example, information about whether this system is weakly-correlated like a closed-shell molecule near equilibrium geometry, or strongly-correlated like an open-shell radical with many quasi-degenerate orbitals. We address this shortcoming by devising the derivative test, Eq. (25), which allows one to tailor the QCC ansatz to a given molecular system in an efficient manner.

We illustrate our approach by considering the dissociation of the $\mathrm{H}_{2}$ and $\mathrm{LiH}$ molecules in the minimal basis set. $\mathrm{LiH}$ contains more orbitals than $\mathrm{H}_{2}$, and hence, more qubits are needed, but what is more important, the quantum entanglement in $\mathrm{LiH}$ is considerably more complex than in $\mathrm{H}_{2}$. Even with this complexity we identified only seven generators of entanglement, Pauli words, that are needed for the QCC parametrization, Eq. (14), to achieve chemical accuracy for the entire ground state curve. This results in only 15 variational parameters - 8 Bloch angles and 7 generators' amplitudes. These numbers are considerably lower, in many cases by few orders of magnitude, than the number of similar quantities used by other methods. We hope, therefore, that the QCC method will become the method of choice for quantum chemistry on a quantum computer in near future.

\section{ACKNOWLEDGMENTS}

A.F.I. acknowledges financial support from Natural Sciences and Engineering Research Council of Canada (NSERC) through the Engage Plus grant.

\section{Appendix: Qubit coupled-cluster energy derivatives}

Let us define $\widetilde{H}(t)=\mathrm{e}^{-\mathrm{i} t \hat{P} / 2} \hat{H} \mathrm{e}^{-\mathrm{i} t \hat{P} / 2}$, then the QCC energy function Eq. (21) can be compactly written as

$$
E(t)=\min _{\boldsymbol{\Omega}}\langle\boldsymbol{\Omega}|\widetilde{H}(t)| \boldsymbol{\Omega}\rangle .
$$

The minimization condition makes the Bloch angles functions of $t$. It is convenient to introduce $\left|\boldsymbol{\Omega}_{\min }\right\rangle$ to denote the coherent state evaluated at optimized Bloch angles, $\left\{\varphi_{i}^{0}\right\}_{i=1}^{2 N_{q}}$.

The first derivative of the similarity transformed energy can be written as

$$
\begin{aligned}
\left.\frac{\mathrm{d} E(t)}{\mathrm{d} t}\right|_{t=0} & =\left\langle\frac{\mathrm{d} \boldsymbol{\Omega}_{\min }}{\mathrm{d} t}|\hat{H}| \boldsymbol{\Omega}_{\min }\right\rangle+\left\langle\boldsymbol{\Omega}_{\min }|\hat{H}| \frac{\mathrm{d} \boldsymbol{\Omega}_{\min }}{\mathrm{d} t}\right\rangle \\
& +\left\langle\boldsymbol{\Omega}_{\min }\left|\frac{\partial \widetilde{H}}{\partial t}\right|_{t=0} \mid \boldsymbol{\Omega}_{\text {min }}\right\rangle .
\end{aligned}
$$

Expanding the derivative of the mean-field state
$\mathrm{d}\left|\boldsymbol{\Omega}_{\min }\right\rangle / \mathrm{d} t$ as

$$
\frac{\mathrm{d}\left|\boldsymbol{\Omega}_{\min }\right\rangle}{\mathrm{d} t}=\sum_{i=1}^{2 N_{q}} \frac{\partial\left|\boldsymbol{\Omega}_{\min }\right\rangle}{\partial \varphi_{i}} \frac{\mathrm{d} \varphi_{i}}{\mathrm{~d} t},
$$

and accounting for the fact that all $\varphi_{i}$ are real, we can write

$$
\begin{aligned}
& \left\langle\frac{\mathrm{d} \boldsymbol{\Omega}_{\min }}{\mathrm{d} t}|\hat{H}| \boldsymbol{\Omega}_{\text {min }}\right\rangle+\left\langle\boldsymbol{\Omega}_{\min }|\hat{H}| \frac{\mathrm{d} \boldsymbol{\Omega}_{\min }}{\mathrm{d} t}\right\rangle \\
& =\sum_{i=1}^{2 N_{q}} \frac{\mathrm{d} \varphi_{i}}{\mathrm{~d} t}\left[\left\langle\frac{\partial \boldsymbol{\Omega}_{\min }}{\partial \varphi_{i}}|\hat{H}| \boldsymbol{\Omega}_{\min }\right\rangle+\left\langle\boldsymbol{\Omega}_{\min }|\hat{H}| \frac{\partial \boldsymbol{\Omega}_{\min }}{\partial \varphi_{i}}\right\rangle\right] \\
& =0 .
\end{aligned}
$$

The sum of derivatives in Eq. (A.4) vanishes because the expression in square brackets is equal to 0 for any $i$ due to the condition of $\varphi_{i}$ optimality. Thus, Eq. (25) follows from Eq. (A.2) as

$$
\left.\frac{\partial \widetilde{H}}{\partial t}\right|_{t=0}=-\frac{\mathrm{i}}{2}[\hat{H}, \hat{P}] .
$$

To derive an expression for the second derivative, consider the QCC energy as a function of entangler's amplitudes and Bloch angles [cf. Eq. (18)]

$$
E_{e}(t, \varphi)=\langle\boldsymbol{\Omega}|\widetilde{H}(t)| \boldsymbol{\Omega}\rangle .
$$

Expanding $E_{e}$ into a multivariate Taylor series at $t=0$ and $\varphi_{i}=\varphi_{i}^{0}$ up to the second order gives

$$
\begin{aligned}
E_{e}(t, \boldsymbol{\varphi}) & =E_{\mathrm{QMF}}+\left.t \frac{\partial E_{e}}{\partial t}\right|_{\substack{t=0 \\
\varphi_{i}=\varphi_{i}^{0}}}+\left.\frac{t^{2}}{2} \frac{\partial^{2} E_{e}}{\partial t^{2}}\right|_{\substack{t=0 \\
\varphi_{i}=\varphi_{i}^{0}}} \\
& +t \mathbf{c}^{T} \cdot \Delta \boldsymbol{\varphi}+\frac{1}{2} \Delta \boldsymbol{\varphi}^{T} \mathcal{D} \Delta \boldsymbol{\varphi},
\end{aligned}
$$

where $\Delta \varphi=\varphi-\varphi^{0}$

$$
\begin{gathered}
c_{i}=\left.\frac{\partial^{2} E_{e}}{\partial t \partial \varphi_{i}}\right|_{\substack{t=0 \\
\varphi_{i}=\varphi_{i}^{0}}}, \\
D_{i j}=\left.\frac{\partial^{2} E_{e}}{\partial \varphi_{i} \partial \varphi_{j}}\right|_{\substack{t=0 \\
\varphi_{i}=\varphi_{i}^{0}}},
\end{gathered}
$$

and the first partial derivatives $\partial E_{e} / \partial \varphi$ at $t=0$ are zero because we have chosen the origin of the Tailor expansion at the mean-field solution. In fact, Bloch angles are minimized in Eq. (A.1) for every value of the amplitude $t$, not only at $t=0$, which leads to the following linear equations that determine the optimal set of Bloch angles for a given $t$

$$
\mathbf{0}=\frac{\partial E_{e}}{\partial \varphi}=t \mathbf{c}+\mathcal{D} \Delta \varphi
$$

or

$$
\Delta \varphi=-t \mathcal{D}^{-1} \mathbf{c}
$$


Equation (A.11) can be interpreted as a linearized form of a nonlinear equation that determines minimizing Bloch angles as functions of the amplitude in Eq. (21).

Inserting Eq. (A.11) into Eq. (A.7) and collecting terms of the same power in $t$ gives the second-order derivative of $E(t)$ in terms of derivatives in Eq. (A.7)

$$
\left.\frac{\mathrm{d}^{2} E_{e}(t)}{\mathrm{d} t^{2}}\right|_{t=0}=\left.\frac{\partial^{2} E_{e}}{\partial t^{2}}\right|_{\substack{t=0 \\ \varphi_{i}=\varphi_{i}^{0}}}-\mathbf{c}^{T} \mathcal{D}^{-1} \mathbf{c}
$$

General expressions for the derivatives in the right-hand side of Eq. (A.12) follow

$$
\begin{aligned}
\left.\frac{\partial^{2} E_{e}}{\partial t^{2}}\right|_{\substack{t=0 \\
\varphi_{i}=\varphi_{i}^{0}}} & =\left\langle\boldsymbol{\Omega}_{\min }\left|\frac{\partial^{2} \widetilde{H}}{\partial t^{2}}\right|_{t=0} \mid \boldsymbol{\Omega}_{\min }\right\rangle \\
& =\left\langle\boldsymbol{\Omega}_{\min }\left|\frac{1}{2} \hat{P}[\hat{H}, \hat{P}]\right| \boldsymbol{\Omega}_{\min }\right\rangle
\end{aligned}
$$

and

$$
\begin{aligned}
c_{i}= & \left.\frac{\partial^{2} E_{e}}{\partial t \partial \varphi_{i}}\right|_{\substack{t=0 \\
\varphi_{i}=\varphi_{i}^{0}}}=\frac{\partial}{\partial \varphi_{i}}\left(\frac{\partial E_{e}}{\partial t}\right) \\
= & \left\langle\frac{\partial \boldsymbol{\Omega}_{\min }}{\partial \varphi_{i}}\left|-\frac{\mathrm{i}}{2}[\hat{H}, \hat{P}]\right| \boldsymbol{\Omega}_{\min }\right\rangle \\
& +\left\langle\boldsymbol{\Omega}_{\min }\left|-\frac{\mathrm{i}}{2}[\hat{H}, \hat{P}]\right| \frac{\partial \boldsymbol{\Omega}_{\min }}{\partial \varphi_{i}}\right\rangle, \\
D_{i j}= & \left\langle\frac{\partial^{2} \boldsymbol{\Omega}_{\min }}{\partial \varphi_{i} \partial \varphi_{j}}|\hat{H}| \boldsymbol{\Omega}_{\min }\right\rangle+\left\langle\frac{\partial \boldsymbol{\Omega}_{\min }}{\partial \varphi_{i}}|\hat{H}| \frac{\partial \boldsymbol{\Omega}_{\min }}{\partial \varphi_{j}}\right\rangle \\
& +\left\langle\frac{\partial \boldsymbol{\Omega}_{\min }}{\partial \varphi_{j}}|\hat{H}| \frac{\partial \boldsymbol{\Omega}_{\min }}{\partial \varphi_{i}}\right\rangle+\left\langle\boldsymbol{\Omega}_{\min }|\hat{H}| \frac{\partial^{2} \boldsymbol{\Omega}_{\min }}{\partial \varphi_{i} \partial \varphi_{j}}\right\rangle .
\end{aligned}
$$

To evaluate the derivatives of the coherent states, Eq. (16), that appear in Eqs. (A.14) and (A.15). Since the coherent states constitute a full basis in the (twodimensional) Hilbert space of a single qubit, it is possible to express the corresponding derivatives in the form of operators acting on a given coherent state. In particular, if $\theta_{i}$ is a polar angle of the $i^{t h}$ qubit, then direct evaluation of the corresponding derivative from Eq. (16) gives

$$
\frac{\partial\left|\Omega_{i}\right\rangle}{\partial \theta_{i}}=\frac{1}{2}\left[-\sin \left(\frac{\theta_{i}}{2}\right)|\alpha\rangle+\mathrm{e}^{\mathrm{i} \phi_{i}} \cos \left(\frac{\theta_{i}}{2}\right)|\beta\rangle\right] .
$$

This derivative can be also written as

$$
\begin{aligned}
\frac{\partial\left|\Omega_{i}\right\rangle}{\partial \theta_{i}} & =\frac{1}{2}\left[-\mathrm{e}^{-\mathrm{i} \phi_{i}} \hat{s}_{+}(i)+\mathrm{e}^{\mathrm{i} \phi_{i}} \hat{s}_{-}(i)\right]\left|\Omega_{i}\right\rangle \\
& =\frac{\mathrm{i}}{2}\left[\hat{x}_{i} \sin \left(\phi_{i}\right)-\hat{y}_{i} \cos \left(\phi_{i}\right)\right]\left|\Omega_{i}\right\rangle,
\end{aligned}
$$

where $\hat{s}_{+}(i)=\left(\hat{x}_{i}+\mathrm{i} \hat{y}_{i}\right) / 2, \hat{s}_{-}(i)=\left(\hat{x}_{i}-\mathrm{i} \hat{y}_{i}\right) / 2, \hat{x}_{i}$, and $\hat{y}_{i}$ are spin raising, lowering, Pauli $\hat{x}$, and $\hat{y}$ operators for the $i^{\text {th }}$ qubit, respectively. Analogously, the derivative of $\left|\Omega_{i}\right\rangle$ with respect to the azimuthal angle $\phi_{i}$ can be evaluated as

$$
\begin{aligned}
\frac{\partial\left|\Omega_{i}\right\rangle}{\partial \phi_{i}} & =\mathrm{i} \sin \left(\frac{\theta_{i}}{2}\right) \mathrm{e}^{\mathrm{i} \phi_{i}}|\beta\rangle \\
& =\mathrm{ie}^{\mathrm{i} \phi_{i}} \tan \left(\frac{\theta_{i}}{2}\right) \hat{s}_{-}(i)\left|\Omega_{i}\right\rangle .
\end{aligned}
$$

Successive application of Eqs. (A.17) and (A.18) gives access to higher-order derivatives of the coherent states.

Determining missing angles: From Eq. (16) it is obvious that values of $\phi_{i}$ angles corresponding to $\theta_{i}=0$ or $\pi$ will not affect qubit mean field energy, and thus, the mean-field procedure does not define them. However, these angles will determine the values of the second derivatives due to differentiation in Eq. (A.17). To determine these $\phi_{i}$ angles we re-optimize all angles for small values of $\tau$ (e.g. $10^{-3}$ ) in the similarity transformed Hamiltonian. This procedure can be combined with a derivative discontinuity test that we discuss next.

Derivative discontinuities: Another caveat of working with the derivatives of similarity transformed energies Eq. (A.1) is that occasionally, first derivatives have discontinuities in the $\tau=0$ point. In this case, the second derivative is not defined and should not be used. Such situations can be detected by evaluating QMF solutions for the similarity transformed Hamiltonian [Eq. (20)] with small amplitudes (e.g. $\tau= \pm 10^{-3}$ ) and calculating the first derivatives in these points using Eq. (25) with the $\hat{H}[\tau ; \hat{P}]$ Hamiltonian instead of $\hat{H}$. If the first derivatives are significantly different, this is a numerical indication of the derivative discontinuity, and the second derivative is not evaluated.
1 S. Lloyd, Science 273, 1073 (1996).

2 A. Aspuru-Guzik, A. D. Dutoi, P. J. Love, and M. HeadGordon, Science 309, 1704 (2005).

3 T. Helgaker, P. Jorgensen, and J. Olsen, Molecular Electronic-structure Theory (Wiley, 2000).

4 P. Jordan and E. Wigner, Z. Phys. 47, 631 (1928).

5 S. B. Bravyi and A. Y. Kitaev, Ann. Phys. 298, 210 (2002).
6 J. T. Seeley, M. J. Richard, and P. J. Love, J. Chem. Phys. 137, 224109 (2012).

7 A. Tranter, S. Sofia, J. Seeley, M. Kaicher, J. McClean, R. Babbush, P. V. Coveney, F. Mintert, F. Wilhelm, and P. J. Love, Int. J. Quantum Chem. 115, 1431 (2015).

8 K. Setia and J. D. Whitfield, ArXiv e-prints (2017), arXiv:1712.00446 [quant-ph]. 
9 V. Havlíček, M. Troyer, and J. D. Whitfield, Phys. Rev. A 95, 032332 (2017).

10 D. S. Abrams and S. Lloyd, Phys. Rev. Lett. 79, 2586 (1997).

11 D. S. Abrams and S. Lloyd, Phys. Rev. Lett. 83, 5162 (1999).

12 M. Nielsen and I. Chuang, Quantum Computation and Quantum Information: 10th Anniversary Edition (Cambridge University Press, 2010).

13 A. Peruzzo, J. McClean, P. Shadbolt, M.-H. Yung, X.-Q. Zhou, P. J. Love, A. Aspuru-Guzik, and J. L. O'Brien, Nat. Commun. 5, 4213 (2014).

14 D. Wecker, M. B. Hastings, and M. Troyer, Phys. Rev. A 92, 042303 (2015).

15 A. G. Taube and R. J. Bartlett, Int. J. Quantum Chem. 106, 3393 (2006).

16 J. Olsen, J. Chem. Phys. 113, 7140 (2000).

17 P. J. J. O'Malley, R. Babbush, I. D. Kivlichan, J. Romero, J. R. McClean, R. Barends, J. Kelly, P. Roushan, A. Tranter, N. Ding, B. Campbell, Y. Chen, Z. Chen, B. Chiaro, A. Dunsworth, A. G. Fowler, E. Jeffrey, E. Lucero, A. Megrant, J. Y. Mutus, M. Neeley, C. Neill, C. Quintana, D. Sank, A. Vainsencher, J. Wenner, T. C. White, P. V. Coveney, P. J. Love, H. Neven, A. Aspuru-Guzik, and J. M. Martinis, Phys. Rev. X 6, 031007 (2016).

18 J. D. Whitfield, J. Biamonte, and A. Aspuru-Guzik, Mol. Phys. 109, 735 (2011).

19 J. Romero, R. Babbush, J. McClean, C. Hempel, P. Love, and A. Aspuru-Guzik, Quantum Sci. Technol. (2018), 10.1088/2058-9565/aad3e4.

20 P. K. Barkoutsos, J. F. Gonthier, I. Sokolov, N. Moll, G. Salis, A. Fuhrer, M. Ganzhorn, D. J. Egger, M. Troyer, A. Mezzacapo, S. Filipp, and I. Tavernelli, Physical Review A 98, 022322 (2018).

21 A. Kandala, A. Mezzacapo, K. Temme, M. Takita, M. Brink, J. M. Chow, and J. M. Gambetta, Nature 549, 242 (2017).

22 E. Rieffel and W. Polak, "Quantum computing: A gentle introduction," (The MIT Press, 2011) p. 89.

23 J. M. Radcliffe, J. Phys. A. 4, 313 (1971).

24 F. T. Arecchi, E. Courtens, R. Gilmore, and H. Thomas, Phys. Rev. A 6, 2211 (1972).

25 A. Perelomov, Generalized Coherent States and Their Applications, Theoretical and Mathematical Physics (Springer Science \& Business Media, 2012).

26 E. H. Lieb, Commun. Math. Phys. 31, 327 (1973).

27 P. Lykos and G. W. Pratt, Rev. Mod. Phys. 35, 496 (1963).

28 I. G. Ryabinkin, S. N. Genin, and A. F. Izmaylov, "Constrained variational quantum eigensolver: Quantum computer search engine in the Fock space," (2018), arXiv:1806.00461 [physics.chem-ph].

29 C. Hempel, C. Maier, J. Romero, J. McClean, T. Monz, H. Shen, P. Jurcevic, B. P. Lanyon, P. Love, R. Babbush, A. Aspuru-Guzik, R. Blatt, and C. F. Roos, Phys. Rev. X 8, 031022 (2018).

30 M. S. Gordon and M. W. Schmidt, in Theory and Applications of Computational Chemistry. The first forty years, edited by C. E. Dykstra, G. Frenking, K. S. Kim, and G. E. Scuseria (Elsevier, Amsterdam, 2005) pp. 1167-1189, version from May 2013.

31 K. L. Schuchardt, B. T. Didier, T. Elsethagen, L. Sun, V. Gurumoorthi, J. Chase, J. Li, and T. Windus, J. Chem. Inf. Model. 47, 1045 (2007).

32 The full molecular symmetry group is $D_{\infty h}$ and $C_{\infty v}$, but the maximal Abelian subgroups with all-real irreducible representations were chosen instead for the sake of implementation simplicity.

33 M. A. Nielsen, School of Physical Sciences The University of Queensland (2005).

34 J. S. Otterbach, R. Manenti, N. Alidoust, A. Bestwick, M. Block, B. Bloom, S. Caldwell, N. Didier, E. Schuyler Fried, S. Hong, P. Karalekas, C. B. Osborn, A. Papageorge, E. C. Peterson, G. Prawiroatmodjo, N. Rubin, C. A. Ryan, D. Scarabelli, M. Scheer, E. A. Sete, P. Sivarajah, R. S. Smith, A. Staley, N. Tezak, W. J. Zeng, A. Hudson, B. R. Johnson, M. Reagor, M. P. da Silva, and C. Rigetti, ArXiv e-prints (2017), arXiv:1712.05771 [quant-ph].

35 R. S. Smith, M. J. Curtis, and W. J. Zeng, "A practical quantum instruction set architecture," (2016). 